\title{
Antiplatelet therapy with or without PPIs for the secondary prevention of cardiovascular diseases in patients at high risk of upper gastrointestinal bleeding: A systematic review and meta-analysis
}

\author{
WENFA WU ${ }^{1 *}$, JIA LIU $^{2 *}$, HUIWEN YU ${ }^{1}$ and ZUOFENG JIANG ${ }^{1}$ \\ ${ }^{1}$ Department of General Practice, Guangzhou Red Cross Hospital, Medical College, Jinan University; \\ ${ }^{2}$ Department of VIP Medical Center, the Third Affiliated Hospital, Medical College, \\ Sun Yat-sen University, Guangzhou, Guangdong 510220, P.R. China
}

Received May 21, 2019; Accepted October 4, 2019

DOI: $10.3892 /$ etm.2020.8657

\begin{abstract}
Recurrent upper gastrointestinal (UGI) and cardiovascular (CV) events of the three antiplatelet therapies in patients with cardiovascular diseases (CVD) were compared. Studies published in the PubMed, Embase, and Cochrane Central Register of Controlled Trials electronic databases that compared differences in adverse outcomes associated with the three antiplatelet therapies were reviewed. Five studies with a total number of 7,399 patients were included. No significant differences were found in the incidence of recurrent UGI events among the three antiplatelet therapies. However, in the aspirin-induced ulcer bleeding subgroups, aspirin plus proton pump inhibitors (PPIs) was associated with a significantly lower risk of recurrent UGI events (OR: 0.06, 95\% CI: $0.01-$ $0.32 ; \mathrm{z}=3.30$ and $\mathrm{P}=0.001)$ and UGI bleeding (OR: $0.06,95 \%$ CI: $0.01-0.34 ; \mathrm{z}=3.24$ and $\mathrm{P}=0.001)$ compared to clopidogrel alone. Both aspirin plus PPIs (OR: 2.12, 95\% CI: 1.58-2.84; $\mathrm{z}=5.00$ and $\mathrm{P}<0.01)$ and clopidogrel plus PPIs (OR: 2.57, 95\% CI: $1.89-3.51 ; \mathrm{z}=5.97$ and $\mathrm{P}<0.01$ ) were related to a comparatively higher risk of recurrent $\mathrm{CV}$ events when compared to clopidogrel alone. In patients at high UGI bleeding risk (regardless of whether it was aspirin-induced) and under treatment of single antiplatelet therapy, aspirin plus PPIs should be considered as the first choice for UGI protection rather than clopidogrel alone and clopidogrel plus PPIs. However, in terms
\end{abstract}

Correspondence to: Dr Wenfa Wu, Department of General Practice, Guangzhou Red Cross Hospital, Medical College, Jinan University, 396 Tongfuzhong Road, Haizhu, Guangzhou, Guangdong 510220, P.R. China

E-mail:wenfa6699@163.com

${ }^{*}$ Contributed equally

Key words: clopidogrel, aspirin, proton pump inhibitors, upper gastrointestinal events, cardiovascular events of $\mathrm{CV}$ protection, clopidogrel alone appears to be superior in reducing $\mathrm{CV}$ risk, while clopidogrel plus PPIs may relate to an increased $\mathrm{CV}$ risk due to the potential drug-drug interaction.

\section{Introduction}

Platelet activation and aggregation play a significant role in the pathogenesis of arterial thrombosis and are directly correlated with ischemic events, such as acute coronary syndromes (ACS), stroke, transient ischemic attack (TIA) and peripheral arterial diseases (PAD). Antiplatelet therapy in the form of low-dose aspirin and clopidogrel are the most commonly used drugs and the core and cornerstone of the management of CVD. However, the association of aspirin with gastrointestinal (GI) adverse effects (ranging from peptic ulcer to fatal bleeding or perforation) is well known (1).

The CAPRIE (2) (Clopidogrel versus Aspirin in Patients at Risk of Ischaemic Events) study showed that fewer GI adverse events were found in the clopidogrel group than in the aspirin group. Based on the CAPRIE study, the American College of Cardiology/American Heart Association (ACC/AHA) issued the recommendation that clopidogrel was the optimal choice for patients with CVD who had major GI intolerance to aspirin (primarily those with recent ulcer bleeding) (3). However, further studies found that $12 \%$ of patients who took clopidogrel with a history of ulcer and $15 \%$ of those who took aspirin underwent recurrent GI bleeding within one year $(4,5)$. For patients at high GI risk who have previous major upper gastrointestinal (UGI) complications of peptic ulcer, UGI bleeding or perforation and who are under treatment of ongoing antiplatelet therapy, the optimal treatment strategy remains uncertain.

The conclusion that proton-pump inhibitor (PPI) reduces the rate of recurrent GI bleeding in high GI risk patients who receive aspirin has been confirmed by randomized controlled trials (5). As revealed by Chan et al (6) and Lai et al (7), aspirin in combination with esomeprazole was superior to clopidogrel for preventing ulcer complications in patients who had previous aspirin-related peptic ulcer bleeding. However, 
in a recent observational study, Tsai et al (8) suggested that clopidogrel alone and clopidogrel plus PPIs were both related to lower risk of GI events than aspirin plus PPIs. Apart from the risk of $\mathrm{CV}$ events, clopidogrel alone was superior to aspirin plus PPIs, while clopidogrel plus PPIs was associated with a significantly higher risk than aspirin plus PPIs.

Hsu et al (9) showed that clopidogrel plus esomeprazole was superior to clopidogrel alone in the prevention of recurrent peptic ulcer in patients with previous peptic ulcer, while there were no differences in CV events between the two groups. In addition, it was reported that aspirin plus PPIs was related to a lower risk of recurrent hospitalization for major GI complications albeit this benefit was not evident in the clopidogrel plus PPIs group (10).

The abovementioned controversial results and data need confirmation through a meta-analysis by comparing the recurrent UGI and $\mathrm{CV}$ events of three antiplatelet therapies: clopidogrel alone, clopidogrel plus PPIs and aspirin plus PPIs.

\section{Materials and methods}

Data sources and search strategy. Studies published in the EMbase, PubMed and Cochrane Central Register of Controlled Trials electronic databases were reviewed. To identify relevant studies, the references of relevant articles were also searched. All relevant articles published from January, 1974 to February, 2018 were chosen. Keywords used for the searches were 'antiplatelet or aspirin or clopidogrel', 'recurrent or recurrence or relapse', 'gastrointestinal hemorrhage or gastrointestinal bleeding', 'ulcer or perforation' and 'proton pump inhibitors or proton pump inhibitor or omeprazole or pantoprazole or rabeprazole or esomeprazole or lansoprazole' in different combinations. There was no limit on sample size, sex or the location of the original study. Only English articles were chosen.

Inclusion criteria. Studies were included if they were randomized controlled trials or observational studies comparing three antiplatelet therapies - clopidogrel, clopidogrel plus PPIs and aspirin plus PPIs - for the secondary prevention of CVD in patients with a history of major UGI complications of peptic ulcer, UGI bleeding or perforation. Studies were included if they reported adverse outcomes (at least recurrent UGI events) as the clinical endpoints, or involved relevant data that could be used in this analysis.

Exclusion criteria. Studies were excluded if they were systematic reviews, meta-analyses, case studies or letters to the editor; if they did not include patients with CVD; if they included patients who used a combination of aspirin and clopidogrel; if they included patients who used a combination of nonsteroidal anti-inflammatory drugs (NSAIDs), anticoagulant agents, cyclooxygenase-2 inhibitors, other antiplatelet drugs or corticosteroids; if they did not report the previously mentioned clinical outcomes; or if they were associated with the same trial or cohort or they were duplicates of the same study.

Types of participants. Patients were identified as having a record of the following indications: coronary heart disease (CHD), PAD, ischemic stroke or TIA and a history of major UGI complications of peptic ulcer, UGI bleeding (including patients with UGI bleeding while receiving low-dose aspirin) or perforation. The participants initiated single antiplatelet therapy by aspirin or clopidogrel for the secondary prevention of cardiovascular events.

Outcomes and follow-up. The primary outcomes were recurrent UGI events which were defined as a hospitalization with the primary diagnosis of UGI bleeding or ulcer (gastric ulcer, duodenal ulcer or peptic ulcer) or perforation and the secondary outcomes were at least one of the following variables: CV events, overall mortality or vascular death. CV events were defined as either a hospitalization due to any of the following: CHD, myocardial infarction (MI), unstable angina (UA), congestive heart failure (CHF), PAD, cerebrovascular insufficiency (CI), ischemic stroke or TIA. The follow-up time extended until the first occurrence of outcomes or for those who did not experience any outcome until the end of the study. All analyses were based on the previously published studies. Therefore, there was no need for ethics approval and patient consent. These outcomes and follow-up periods are summarized in Table I.

Data extraction and quality assessment. Information and data, including the names of the authors, country or region of the study, year of article publication, period of patients' enrollment, number of patients in each group (clopidogrel, clopidogrel plus PPIs and aspirin plus PPIs), type of study (RCT or observational study), baseline characteristics of the patients, the outcomes reported, the follow-up periods, the medications used by the patients and the number of events reported for clopidogrel, clopidogrel plus PPIs and aspirin plus PPIs were carefully extracted by two of the authors. Any disagreement was discussed and resolved by consulting a third author.

Since this was a meta-analysis, we followed the PRISMA guideline statement (11). The Cochrane Risk of Bias Tool for Non-Randomized Studies of Interventions (ACROBATNRSI) was used to evaluate the quality and the bias risk of the included trials by two independent reviewers (12). All the trials were rated as having a low risk of bias.

Statistical analysis. The statistical analysis was carried out using Revman 5.3 software with odds ratio (OR) and $95 \%$ confidence intervals (CI) as the analytical parameters. Heterogeneity across the studies was assessed by the Q statistic test and the $\mathrm{I}^{2}$ test (The lower the $\mathrm{I}^{2}$ value, the lower the heterogeneity, whereas heterogeneity would increase with an increasing $\mathrm{I}^{2}$ value) (13). A P-value of $\leq 0.05$ was considered statistically significant. If the $\mathrm{I}^{2}$ value was $>50 \%$, a random effects model was used for the analysis, whereas for an $\mathrm{I}^{2}$ value $<50 \%$, a fixed effects model was used. Forest plot was used to graphically display the results. A funnel plot was used to visually assess the potential publication bias.

\section{Results}

Search results. Fig. 1 shows the selection process for the eligible studies. In total, 173 records were identified using the abovementioned search terms in electronic databases. After removing 143 duplicate records and 11 case-only studies, comments, review articles, animal models or case reports, 
Table I. Reported end points and follow-up periods.

\begin{tabular}{llcl}
\hline Studies (Refs.) & \multicolumn{1}{c}{ Reported end points } & Follow-up period & Types of participants \\
\hline Chan 2005 (6) & $\begin{array}{l}\text { Recurrent ulcer bleeding, MI, UA, CI, } \\
\text { overall mortality, vascular death } \\
\text { Recurrent ulcer bleeding, perforation, } \\
\text { obstruction, MI, CHF, IS, overall } \\
\text { mortality, vascular death }\end{array}$ & 12 months & $\begin{array}{l}\text { Aspirin-induced upper } \\
\text { GI bleeding }\end{array}$ \\
& Recurrent ulcer, ulcer bleeding, perforation & 52 weeks & $\begin{array}{l}\text { Aspirin-induced upper } \\
\text { GI bleeding }\end{array}$ \\
Hsiao 2009 (10) & Recurrent ulcer, ulcer bleeding, & 2 years & Previous peptic ulcer or GI \\
Tsai 2011 (8) & $\begin{array}{l}\text { perforation, CHD, MI, PVD, IS, TIA } \\
\text { Recurrent ulcer, ulcer bleeding, UA, MI, }\end{array}$ & 1 year & $\begin{array}{l}\text { Previous peptic ulcer or } \\
\text { GI bleeding }\end{array}$ \\
Hsu 2011 (9) & IS, overall mortality & 6 months & Previous peptic ulcer \\
\hline
\end{tabular}

MI, myocardial infarction; UA, unstable angina; CI, cerebrovascular insufficiency; CHF, congestive heart failure; IS, ischemic stroke; GI, gastrointestinal; CHD, coronary heart diseases; PVD, peripheral vascular diseases; TIA, transient ischemic attacks.

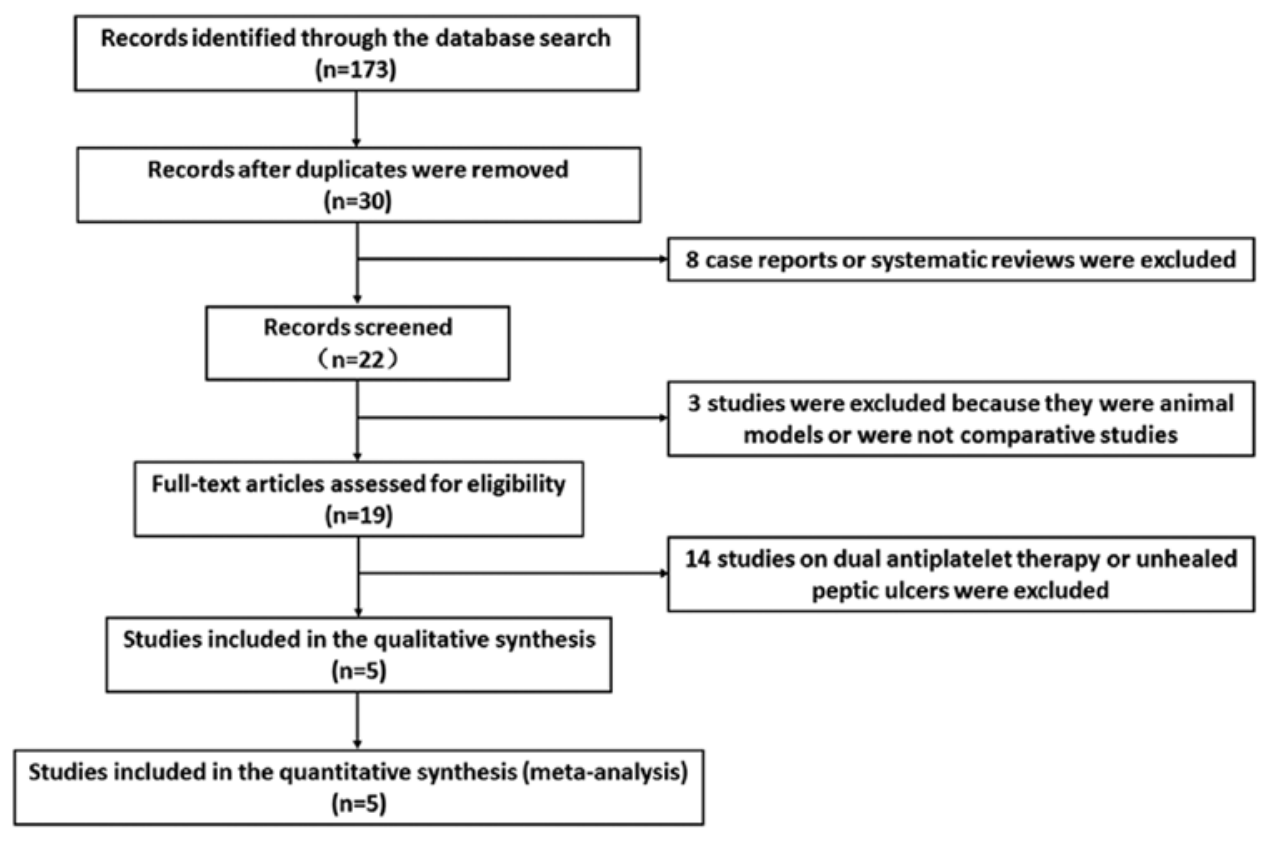

Figure 1. PRISMA flow chart for the selection process of eligible studies.

19 records remained for assessment. Fourteen studies were excluded due to the use of a combination of aspirin and clopidogrel or the inclusion of subjects with unhealed peptic ulcer. Finally, 3 randomized controlled trials $(7,8,10)$ and 2 observational studies $(9,11)$ were included with a total number of 7,399 patients $(3,688$ patients were treated with clopidogrel alone, 1,725 patients were treated with clopidogrel plus PPIs and 1,986 were treated with aspirin plus PPIs). We found that the regions of the included studies comprised only Hong Kong and Taiwan. The general and baseline features of the included studies are listed in Table II.

\section{Aspirin plus PPIs versus clopidogrel}

Recurrent UGI events. In the overall meta-analysis, there was significant heterogeneity $\left(\chi^{2}=58.70, \mathrm{df}=3\right.$ and $\mathrm{P}<0.00001$;
$\mathrm{I}^{2}=95 \%$ ) among the four studies. In the random effects model, however, although recurrent UGI events favored aspirin plus PPIs with OR: $0.75,95 \%$ CI: $0.20-2.85 ; z=0.42$ and $\mathrm{P}=0.68$, the result was not statistically significant (Fig. 2A). Due to the presence of significant heterogeneity among the included studies, the above result may be considered inadequate and biased. The funnel plot showed the minimal publication bias (Fig. 3). The possible reasons might be the included study type (observational) and the selection of only English publications for this analysis.

When randomized data were analyzed separately, in the study by Chan et al (6), 1 case of recurrent duodenal ulcer bleeding and 13 cases of recurrent ulcer bleeding $(6$ gastric ulcers, 5 duodenal ulcers and 2 both gastric and duodenal ulcers) were observed in the aspirin-plus-esomeprazole group 


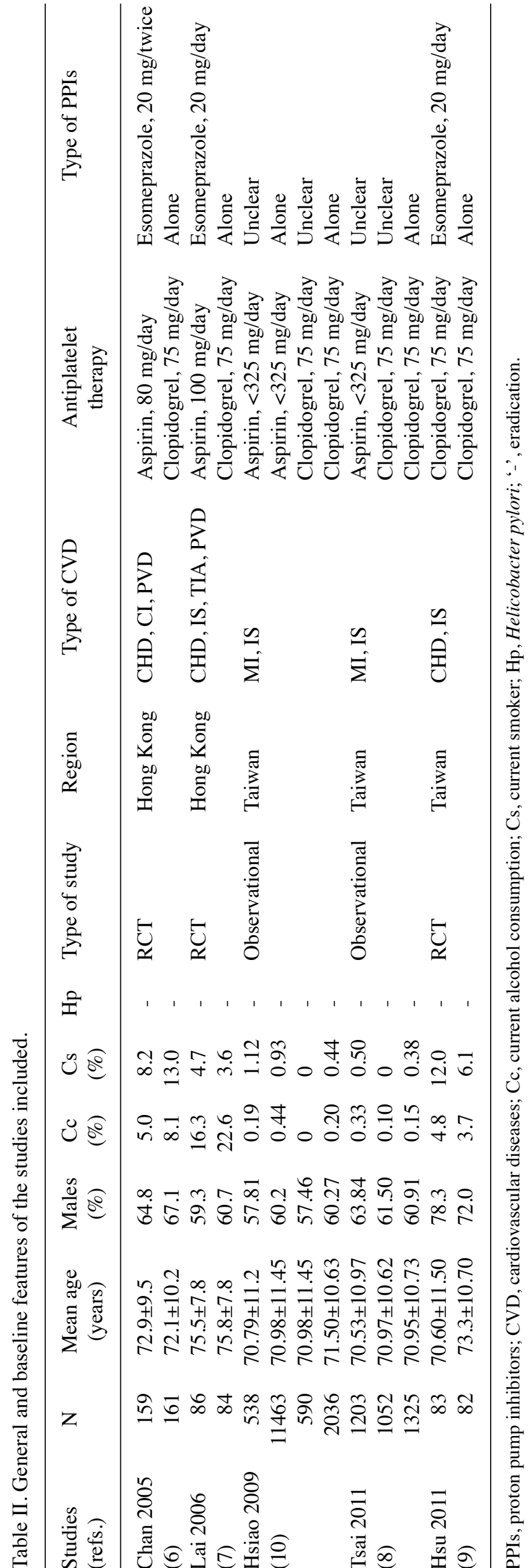

and the clopidogrel group, respectively. Lai et al (7) found that no recurrent ulcer complications occurred in the aspirin plus PPIs group, and 9 cases (8 gastric and/or duodenal ulcers bleeding and 1 perforated duodenal ulcer) occurred in the clopidogrel group. No significant heterogeneity $\left(\chi^{2}=0.06, \mathrm{df}=1\right.$ and $\mathrm{P}=0.80 ; \mathrm{I}^{2}=0 \%$ ) was found between those two studies. In the fixed effects model, recurrent UGI events were significantly lower with aspirin plus PPIs with OR: $0.06,95 \%$ CI: $0.01-0.32 ; \mathrm{z}=3.30$ and $\mathrm{P}=0.001$. This analysis also showed that recurrent UGI bleeding still significantly favored aspirin plus PPIs with OR: $0.06,95 \%$ CI: $0.01-0.34 ; \mathrm{z}=3.24$ and $\mathrm{P}=0.001$ (Fig. 2B).

When observational data were analyzed separately, there was significant heterogeneity $\left(\chi^{2}=44.01, \mathrm{df}=1\right.$ and $\mathrm{P}<0.00001$; $\mathrm{I}^{2}=98 \%$ ) between the two studies. In the random effects model, recurrent UGI events favored clopidogrel with OR: $2.52,95 \%$ CI: $0.58-10.89 ; \mathrm{z}=1.24$ and $\mathrm{P}=0.22$, but the result was not statistically significant (Fig. 2C).

Recurrent $C V$ events. In the overall meta-analysis, no significant heterogeneity $\left(\chi^{2}=1.62, \mathrm{df}=2\right.$ and $\left.\mathrm{P}=0.44 ; \mathrm{I}^{2}=0 \%\right)$ was found among the three studies. In the fixed effects model, the rate of recurrent $\mathrm{CV}$ events was significantly higher with aspirin plus PPIs with OR: $2.12,95 \%$ CI: $1.58-2.84 ; z=5.00$ and $\mathrm{P}<0.00001$ (Fig. 2D). There was no significant publication bias as indicated by the funnel plot (Fig. 4).

When randomized data were separately analyzed in the study by Chan et al (6), recurrent ischemic CV events occurred in 11 patients in the aspirin plus PPIs group (1 MI, 7 UA and $3 \mathrm{CI})$ and in 9 patients in the clopidogrel group (1 MI, $6 \mathrm{UA}$ and $2 \mathrm{CI}$ ). Lai et al (7) found that 1 patient developed CHF, 1 patient developed MI, and 1 patient developed recurrent stroke in the aspirin plus PPIs group, while 1 patient developed MI, and 1 patient developed CHF in the clopidogrel group. No significant heterogeneity $\left(\chi^{2}=0.03, \mathrm{df}=1\right.$, and $\left.\mathrm{P}=0.87 ; \mathrm{I}^{2}=0 \%\right)$ was found between the two studies. The recurrent $\mathrm{CV}$ events were similar between the aspirin plus PPIs and clopidogrel groups with OR: $1.30,95 \%$ CI: $0.58-2.93 ; \mathrm{z}=0.63$ and $\mathrm{P}=0.53$ by the fixed effects model (Fig. 2E).

Overall mortality and vascular death. Of the 12 patients who died in the study reported by Chan et al (6), 4 were in the aspirin plus PPIs group (1 patient died from MI, 1 CI, 1 renal failure and 1 uncertain causes), and 8 were in the clopidogrel group (1 patient died from MI, 1 from an intracranial hemorrhage, 1 from heart failure, 3 from sepsis and 2 from uncertain causes). In the study by Lai et al (7), 3 patients in the aspirin plus PPIs group died of pneumonia, MI and recurrent stroke. In the clopidogrel group, 3 patients died of chronic obstructive airway disease, CHF and chronic renal failure. No significant heterogeneity was found between the two studies. In the fixed effects model, overall mortality and vascular death showed no statistical difference between the aspirin plus PPIs group and the clopidogrel group with OR: $0.63,95 \%$ CI: 0.24-1.64; $\mathrm{z}=0.95$ and $\mathrm{P}=0.34$ and OR: $1.00,95 \%$ CI: $0.25-4.04 ; \mathrm{z}=0.00$ and $\mathrm{P}=1.00$, respectively (Fig. $2 \mathrm{~F}$ ).

Aspirin plus PPIs versus clopidogrel plus PPIs. Two observational studies by Hsiao et al (10) and Tsai et al (8) compared recurrent UGI events between the aspirin plus PPIs group and 


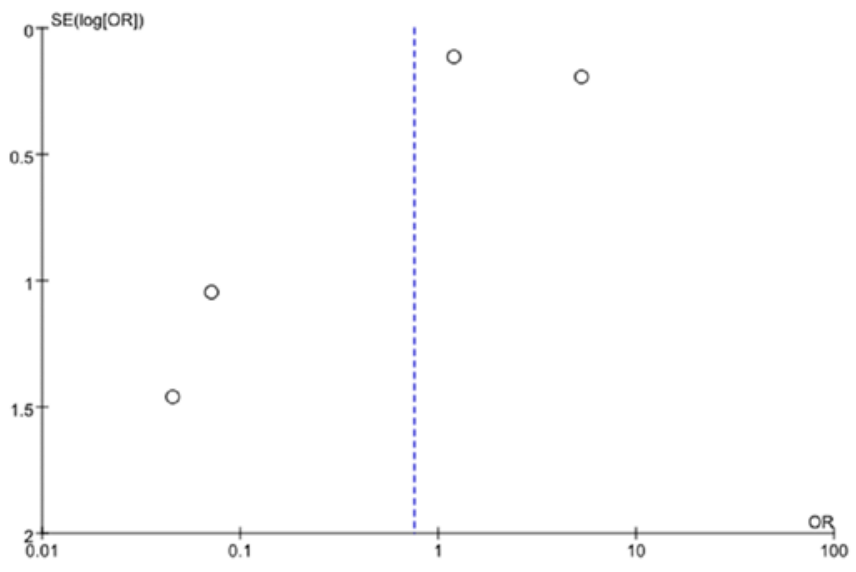

Figure 3. Publication bias assessment. The funnel plot for overall meta-analysis reports on recurrent UGI events between the aspirin plus PPIs and clopidogrel groups. The funnel plot represents minimal publication bias. OR: odds ratio; SE: standard error. UGI, upper gastrointestinal; PPIs, proton pump inhibitors.

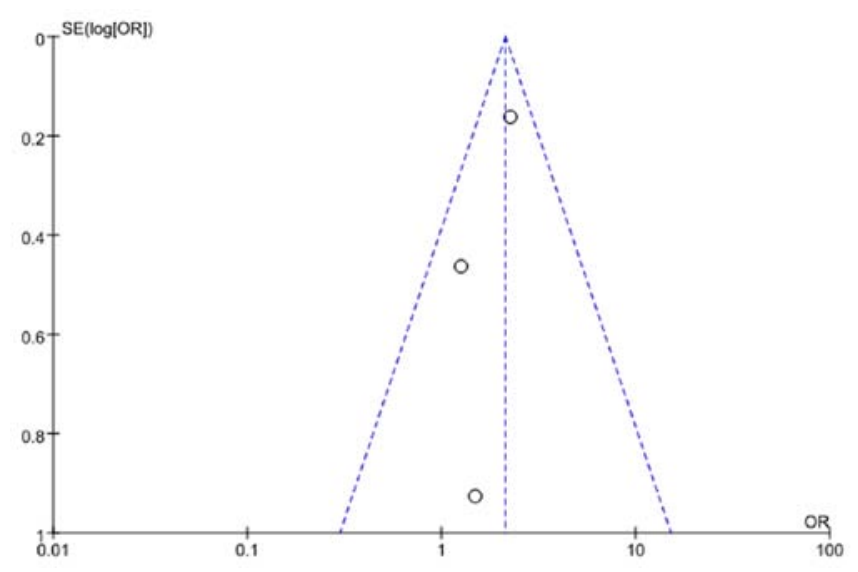

Figure 4. Publication bias assessment. The funnel plot for overall meta-analysis reports on recurrent $\mathrm{CV}$ events between the aspirin plus PPIs and clopidogrel groups. Visual assessment of the funnel plot shows no asymmetry and no obvious publication bias. $\mathrm{CV}$, cardiovascular; PPIs, proton pump inhibitors.

the clopidogrel plus PPIs group. Significant heterogeneity was found between the two studies $\left(\chi^{2}=2.94, \mathrm{df}=1\right.$, and $\mathrm{P}=0.09$; $\left.\mathrm{I}^{2}=66 \%\right)$. In the random effects model, recurrent UGI events were not significantly different with OR: $1.25,95 \% \mathrm{CI}$ : $0.90-1.74 ; \mathrm{z}=1.32$ and $\mathrm{P}=0.19$ (Fig. 5). There were several causes of heterogeneity, including co-medications and comor- bidities (Fig. 5). There was no significant publication bias as indicated by the funnel plot (Fig. 6).

\section{Clopidogrel plus PPIs versus clopidogrel}

Recurrent UGI events. In the overall meta-analysis, significant heterogeneity $\left(\chi^{2}=30.42, \mathrm{df}=2\right.$, and $\mathrm{P}<0.01 ; \mathrm{I}^{2}=93 \%$ ) was found in the three studies. In the random effects model, recurrent UGI events were not significantly different with OR: 1.20, 95\% CI: $0.40-3.65 ; \mathrm{z}=0.33$ and $\mathrm{P}=0.74$ (Fig. 7A). There was no significant publication bias, as indicated by the funnel plot (Fig. 8).

When observational data were separately analyzed, there was significant heterogeneity $\left(\chi^{2}=24.12, \mathrm{df}=1\right.$, and $\mathrm{P}<0.01$; $\mathrm{I}^{2}=96 \%$ ) between the two studies. In the random effects model, recurrent UGI events still showed significant difference with OR: 2.00, 95\% CI: 0.65-6.16; $\mathrm{z}=1.21$ and $\mathrm{P}=0.23$ (Fig. 7B).

Recurrent $C V$ events. No significant heterogeneity $\left(\chi^{2}=0.74\right.$, $\mathrm{df}=1$, and $\mathrm{P}=0.39 ; \mathrm{I}^{2}=0 \%$ ) was found between the two studies. In the fixed effects model, recurrent $\mathrm{CV}$ events were significantly higher with clopidogrel plus PPIs with OR: 2.57, 95\% CI: 1.89-3.51; $\mathrm{z}=5.97$ and $\mathrm{P}<0.01$ (Fig. 7C).

\section{Discussion}

The use of antiplatelet drugs is limited by potential adverse GI complications including peptic ulcer, GI bleeding or perforation, especially in patients with previous GI events. By this analysis, we compared the incidence of recurrent UGI and $\mathrm{CV}$ events in patients at high risk of UGI bleeding who were prescribed one of the three antiplatelet therapies - clopidogrel alone, clopidogrel plus PPIs or aspirin plus PPIs - for the secondary prevention of CVD.

Current results revealed no significant difference in the overall rates of recurrent UGI events between aspirin plus PPIs and clopidogrel alone (Fig. 2A). However, aspirin plus PPIs was associated with a lower risk of recurrent UGI events and UGI bleeding when compared to clopidogrel in subgroups from two randomized studies by Chan et al and by Lai et al $(6,7)$, in which the patients had previous aspirin-induced ulcer bleeding (Fig. 2B). Chan et al (6) studied patients who took aspirin for vascular disease prevention but presented with ulcer bleeding.

After the healing of ulcers, patients who were negative for Helicobacter pylori (Hp) were randomly assigned to receive either $75 \mathrm{mg}$ of clopidogrel daily plus esomeprazole placebo twice daily or $80 \mathrm{mg}$ of aspirin daily plus $20 \mathrm{mg}$ of

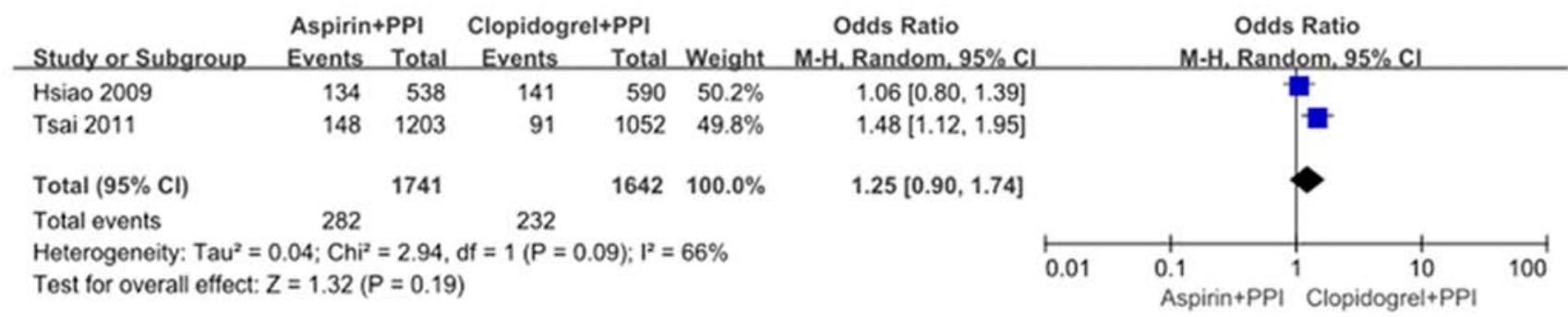

Figure 5. Forest plots showing the relative strength of treatment effects of the aspirin plus PPIs and clopidogrel plus PPIs groups. Two observational studies by Hsiao et al (10) and Tsai et al (8) compare recurrent UGI events between the aspirin plus PPIs group and the clopidogrel plus PPIs group. PPIs, proton pump inhibitors; UGI, upper gastrointestinal. 


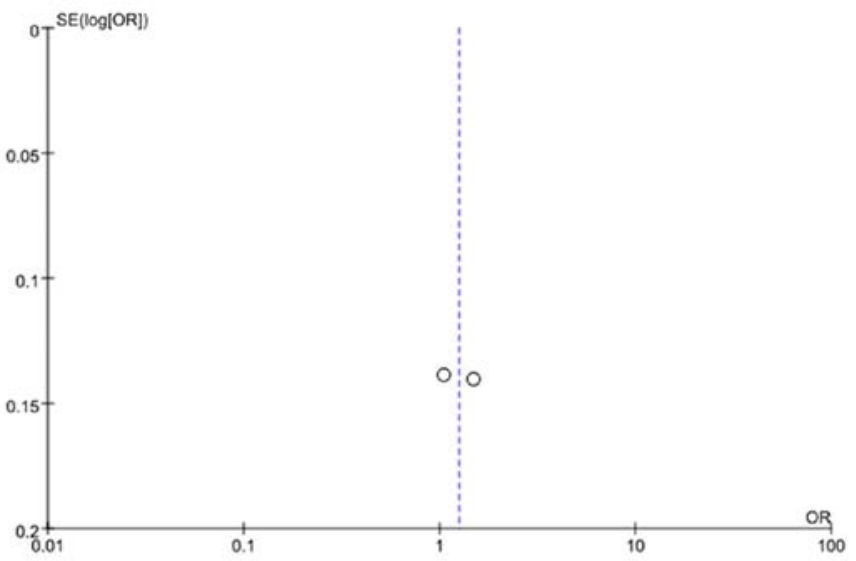

Figure 6. Publication bias assessment. The funnel plot for reports on recurren UGI events between the aspirin plus PPIs and clopidogrel plus PPIs groups. Visual assessment of the funnel plot shows no asymmetry and no obvious publication bias. UGI, upper gastrointestinal; PPIs, proton pump inhibitors.

esomeprazole twice daily for 12 months. A total of 320 patients were enrolled. Recurrent ulcer bleeding occurred in 13 patients receiving clopidogrel and 1 receiving aspirin plus esomeprazole. The cumulative incidence of recurrent bleeding during the 12 -month period was 8.6 and $0.7 \%$, respectively $(\mathrm{P}=0.001)$. Another 170 patients who developed ulcer bleeding after the use of low-dose aspirin were enrolled by Lai et al (7). After the healing of ulcers and the eradication of $\mathrm{Hp}$, the patients were randomly assigned to receive esomeprazole $20 \mathrm{mg}$ /day and aspirin $100 \mathrm{mg} /$ day or clopidogrel $75 \mathrm{mg} /$ day. During a median follow-up period of 52 weeks, no patient in the esomeprazole group developed recurrent ulcer complications, while 9 patients in the clopidogrel group did. The cumulative incidences of recurrent ulcer complications were $0 \%$ and $13.6 \%$, respectively $(\mathrm{P}=0.0019)$. Therefore, we conclude that among patients with previous aspirin-induced ulcer bleeding, aspirin plus PPIs was superior to clopidogrel alone in preventing recurrent ulcer bleeding. A recent large retrospective cohort study by Hsiao et al (10) reported that the mean drug cost per person/ year was several times higher in clopidogrel users than in users of aspirin plus a PPI. Our findings show that clopidogrel is not an ideal substitute for aspirin in patients with previous UGI bleeding, especially aspirin-induced bleeding.

As stated above, $12 \%$ of patients with a previous ulcer who took clopidogrel had recurrent GI bleeding within one year (4). The unclear mechanisms by which clopidogrel led to recurrent ulcer bleeding prompted further studies. Animal studies have shown that platelet adenosine-diphosphate (ADP) receptor antagonists hinder the healing of gastric ulcers by suppressing the releasing of platelet-derived growth factors (14). Therefore, for patients with high GI bleeding risk, clopidogrel alone may not be safe enough and concomitant PPIs prophylaxis is

A

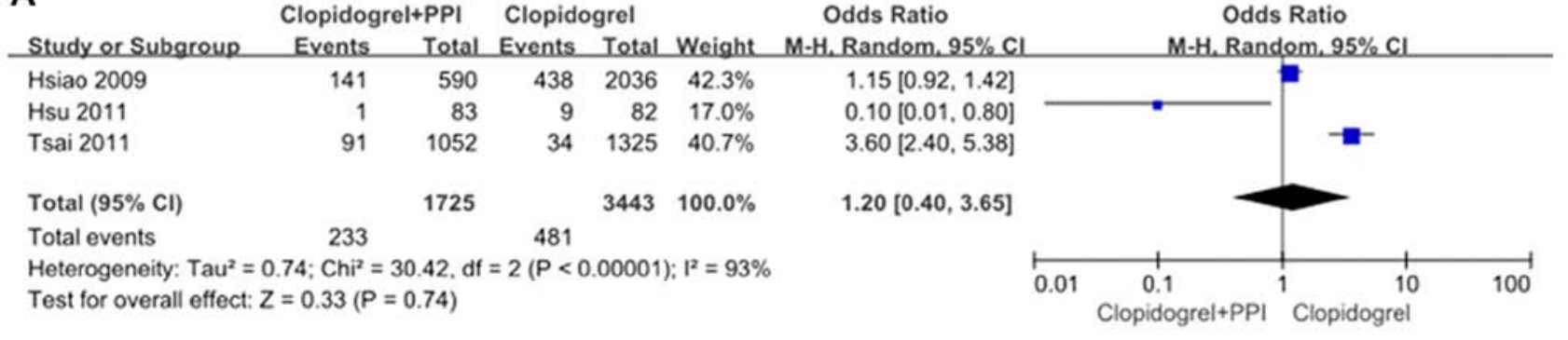

B

\begin{tabular}{|c|c|c|c|c|c|c|c|c|c|c|}
\hline \multirow[b]{2}{*}{ Study or Subgroup } & \multicolumn{2}{|c|}{ Clopidogrel+PPI } & \multicolumn{2}{|c|}{ Clopidogrel } & \multicolumn{2}{|r|}{ Odds Ratio } & \multirow{2}{*}{\multicolumn{4}{|c|}{$\begin{array}{l}\text { Odds Ratio } \\
\text { O. }\end{array}$}} \\
\hline & Events & Total & Events & Total & Weight & M-H, Random, $95 \%$ Cl & & & & \\
\hline Hsiao 2009 & 141 & 590 & 438 & 2036 & $51.1 \%$ & $1.15[0.92,1.42]$ & & & & \\
\hline Tsai 2011 & 91 & 1052 & 34 & 1325 & $48.9 \%$ & $3.60[2.40,5.38]$ & & & & \\
\hline Total $(95 \% \mathrm{Cl})$ & & 1642 & & 3361 & $100.0 \%$ & $2.00[0.65,6.16]$ & & & & \\
\hline Total events & 232 & & 472 & & & & & & & \\
\hline $\begin{array}{l}\text { Heterogeneity: } \mathrm{Tau}^{2} \\
\text { Test for overall effect }\end{array}$ & $\begin{array}{l}63 ; \mathrm{Chi}^{2}= \\
=1.21(\mathrm{P}\end{array}$ & $\begin{array}{l}\text { 12, df } \\
\text {.23) }\end{array}$ & $=1(P<$ & .00001 & $1^{2}=96 \%$ & & 0.01 & 0.1 & 10 & 100 \\
\hline
\end{tabular}

C

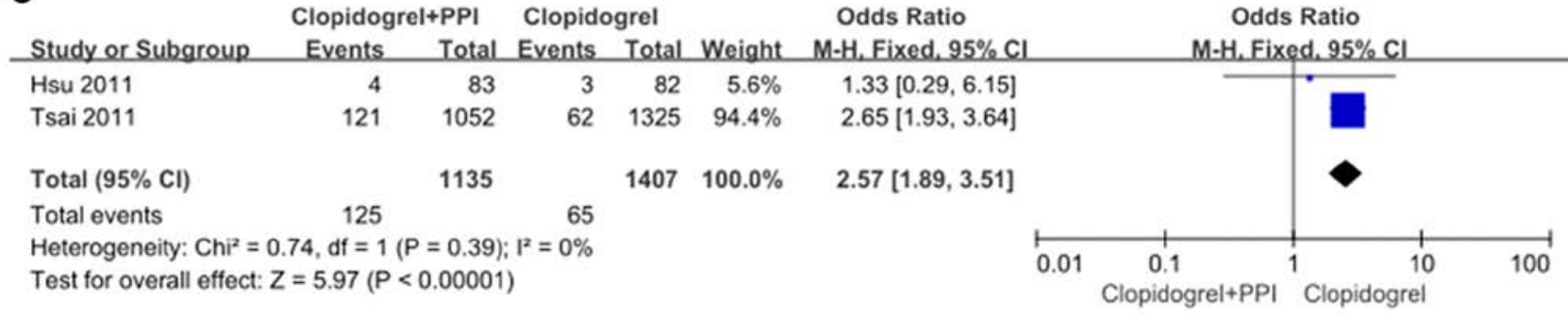

Figure 7. Forest plots showing the relative strength of treatment effects of clopidogrel plus PPIs and clopidogrel groups. The forest plots demonstrate pooled odds ratios between the clopidogrel plus PPIs and clopidogrel groups for (A) recurrent UGI events in the overall meta-analysis, (B) recurrent UGI events using data obtained only from the observational studies of Hsiao et al (10) and Tsai et al (8) and (C) recurrent CV events. PPIs, proton pump inhibitors; UGI, upper gastrointestinal; CV, cardiovascular. 


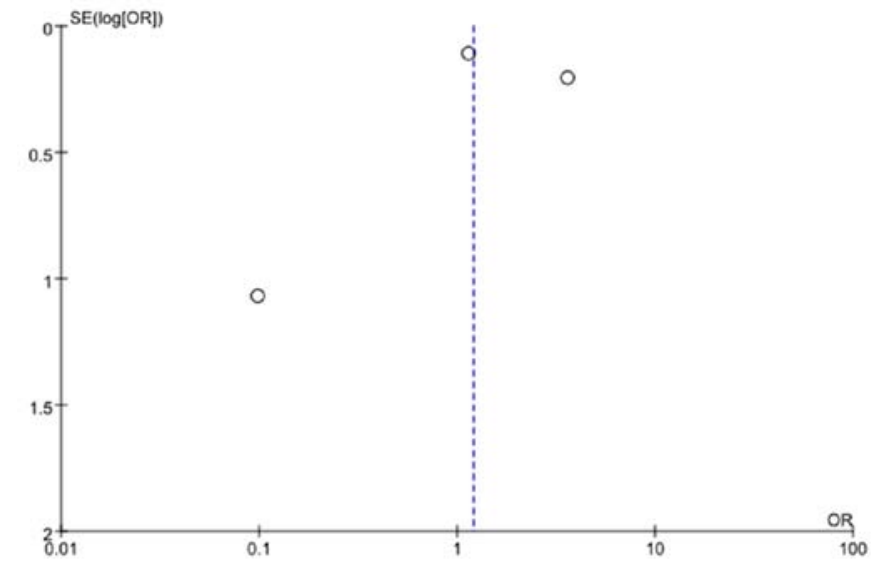

Figure 8. Publication bias assessment. The funnel plot for overall metaanalysis reports on recurrent UGI events between the clopidogrel plus PPIs and clopidogrel groups. Visual assessment of the funnel plot shows no asymmetry and no obvious publication bias. UGI, upper gastrointestinal; PPIs, proton pump inhibitors.

necessary. Whether the concomitant use of clopidogrel and PPIs was superior to aspirin plus PPIs or clopidogrel alone in high GI bleeding risk patients for recurrences of UGI adverse effects is not clear. Our analysis revealed no significant difference in recurrent UGI events when clopidogrel plus PPIs were compared to aspirin plus PPIs and clopidogrel alone (Figs. 5, and 7A and B). Our results were similar to those of the study by $\mathrm{Ng}$ et al (15). Therefore, clopidogrel plus PPIs was not superior to either aspirin plus PPIs or clopidogrel alone for reducing recurrent UGI events in high GI bleeding risk patients.

Nevertheless, aspirin plus PPIs was associated with a significantly higher risk of recurrent $\mathrm{CV}$ events when compared to clopidogrel alone (Fig. 2F). This result might be due to the fact that aspirin inhibits platelet aggregation to a relatively weak extent. Aspirin inhibits thromboxane A2 production by irreversible acetylation of the platelet cyclooxygenase enzyme. Clopidogrel selectively and irreversibly binds to the P2Y12 receptor and inhibits platelet aggregation, thereby blocking the ADP-dependent pathway of platelet activation. Comparative clinical trials suggested that blockade of this pathway may be more powerful and effective than thromboxane A2 inhibition $(16,17)$. Similarly, the CAPRIE (2) trial showed that compared with aspirin, clopidogrel reduced the combined risk of ischaemic stroke, MI or vascular death in high $\mathrm{CV}$ risk patients (by 8.7\%).

However, our analysis showed no significant difference in recurrent $\mathrm{CV}$ events, overall mortality and vascular death in the aspirin-induced ulcer bleeding subgroups when aspirin plus PPIs was compared to clopidogrel alone (Fig. 2E and F).

Furthermore, our analysis showed that clopidogrel plus PPIs was associated with a significantly higher risk of recurrent $\mathrm{CV}$ events when compared to clopidogrel alone (Fig. 7C). Clopidogrel is a prodrug that is converted to an active metabolite in the liver, with the bioactivation mediated by hepatic cytochrome P450 2C19 (18). It was reported that there was a potential cytochrome P450 2C19-dependent drug-drug interaction between clopidogrel and PPIs $(19,20)$. As the competitive inhibitors of cytochrome P450 2C19, PPIs may alter the pharmacokinetics of clopidogrel and potentially lead to a higher risk for recurrent adverse $\mathrm{CV}$ events $(21,22)$. Tsai et al studied a total number of 3,580 patients in the population-based database from Taiwan's National Health Insurance (8). It was included that PPI prophylaxis was associated with a higher risk of CV events in patients who received clopidogrel [with PPI vs. without PPI; HR 2.15 (1.48-3.11)]. Nevertheless, some studies did not show an interaction between clopidogrel and PPIs (22,23). Hsu et al (9) noted that there was no evidence of an interaction between esomeprazole and clopidogrel. As in this study, esomeprazole was administered before breakfast and clopidogrel was given at bedtime. The very short half-lives of PPIs and clopidogrel made this approximately 14-16 h drug administration separation minimize any potential interactions. These findings may provide new ideas for reducing drug interactions.

This analysis is new in several ways. As far as we know, this is the first meta-analysis to compare recurrent UGI and $\mathrm{CV}$ events of three antiplatelet therapies - clopidogrel alone, clopidogrel plus PPIs and aspirin plus PPIs - for the secondary prevention of CVD in patients at high GI bleeding risk. Second, this interesting idea is very important clinically. Third, the randomized and observational studies were combined and were also analyzed separately.

There are several limitations to our meta-analysis. First, there are inherent limitations to an observational study design. As with any non-randomized design, significant differences in comorbidities between different treatment groups could have affected the findings. Second, there was a small sample size of randomized studies included in our meta-analysis when compared to observational studies, and the results might not be very accurate. Third, only 5 studies were included in our meta-analysis, and an assessment of publication bias by a funnel plot may not provide sufficient power to reveal asymmetry. Fourth, the studies included in this meta-analysis are solely from Asia, so the conclusion only applied to the Asia population. A recent meta-analysis suggested the therapeutic effect of PPIs to reduce recurrent bleeding rates after ulcer bleeding was more efficacious in Asia than elsewhere. This may be the result of an enhanced pharmacodynamic effect of PPIs in Asian patients (24). Fifth, we planned to differentiate the risks of the dosage of PPIs, the different PPIs types and the CYP2C19 genotypes for the weakening of clopidogrel efficacy, however, the included studies did not sufficiently report this aspect or did not study this aspect. Therefore, it was impractical to perform an analysis of these variables. Finally, our analysis only discussed single antiplatelet therapy and did not include dual antiplatelet therapy for patients who had experienced prior UGI complications.

In summary, our analysis suggests that in patients at high UGI bleeding risk, whether aspirin-induced or not, who require ongoing single antiplatelet therapy for the secondary prevention of CVD, aspirin plus PPIs may be a more cost-effective option and should be considered as the first choice rather than clopidogrel alone or clopidogrel plus PPIs for UGI protection. However, in terms of CV protection, clopidogrel alone appears to be superior for reducing $\mathrm{CV}$ risk while clopidogrel plus PPIs may be associated with a higher $\mathrm{CV}$ risk due to potential drugdrug interaction in this subset of patients. Further studies are still needed to confirm our results due to the limitations of our meta-analysis. 


\section{Acknowledgements}

Not applicable.

\section{Funding}

No funding was received.

\section{Ethics approval and consent to participate}

Not applicable.

\section{Availability of data and materials}

The datasets used and/or analyzed during the present study are available from the corresponding author on reasonable request.

\section{Authors' contributions}

WW and JL contributed to the study design. ZJ contributed to data extraction and quality assessment. HY was responsible for the analysis and discussion of the data. All authors read and approved the final manuscript.

\section{Patient consent for publication}

Not applicable.

\section{Competing interests}

The authors declare that they have no competing interests.

\section{References}

1. Farrell B, Godwin J, Richards S and Warlow C; UK-TIA Study Group: The United Kingdom transient ischaemic attack (UK-TIA) aspirin trial: Final results. J Neurol Neurosurg Psychiatry 54 1044-1054, 1991.

2. CAPRIE Steering Committee: A randomised, blinded, trial of clopidogrel versus aspirin in patients at risk of ischaemic events (CAPRIE). Lancet 348: 1329-1339, 1996.

3. Braunwald E, Antman EM, Beasley JW, Califf RM, Cheitlin MD, Hochman JS, Jones RH, Kereiakes D, Kupersmith J, Levin TN, Pepine CJ, Schaeffer JW, Smith EE III, Steward DE and Theroux P: ACC/AHA 2002 guideline update for the management of patients with unstable angina and non-ST-segment elevation myocardial infarction: summary article: a report of the American College of Cardiology/American Heart Association Task Force on Practice Guidelines (Committee on the Management of Patients With Unstable Angina). Circulation 106: 1893-1900, 2002.

4. Ng FH, Wong SY, Chang CM, Chen WH, Kng C, Lanas AI and Wong BC: High incidence of clopidogrel-associated gastrointestinal bleeding in patients with previous peptic ulcer disease. Aliment Pharmacol Ther 18: 443-449, 2003.

5. Lai KC, Lam SK, Chu KM, Wong BC, Hui WM, Hu WH, Lau GK, Wong WM, Yuen MF, Chan AO, et al: Lansoprazole for the prevention of recurrences of ulcer complications from long-term low-dose aspirin use. N Engl J Med 346: 2033-2038, 2002.

6. Chan FK, Ching JY, Hung LC, Wong VW, Leung VK, Kung NN, Hui AJ, Wu JC, Leung WK, Lee VW, et al: Clopidogrel versus aspirin and esomeprazole to prevent recurrent ulcer bleeding. $\mathrm{N}$ Engl J Med 352: 238-244, 2005.

7. Lai KC, Chu KM, Hui WM, Wong BC, Hung WK, Loo CK, Hu WH, Chan AO, Kwok KF, Fung TT, et al: Esomeprazole with aspirin versus clopidogrel for prevention of recurrent gastrointestinal ulcer complications. Clin Gastroenterol Hepatol 4: $860-865,2006$.
8. Tsai YW, Wen YW, Huang WF, Chen PF, Kuo KN and Hsiao FY: Cardiovascular and gastrointestinal events of three antiplatelet therapies: Clopidogrel, clopidogrel plus proton-pump inhibitors, and aspirin plus proton-pump inhibitors in patients with previous gastrointestinal bleeding. J Gastroenterol 46: 39-45, 2011.

9. Hsu PI, Lai KH and Liu CP: Esomeprazole with clopidogrel reduces peptic ulcer recurrence, compared with clopidogrel alone, in patients with atherosclerosis. Gastroenterology 140: 791-798, 2011.

10. Hsiao FY, Tsai YW, Huang WF, Wen YW, Chen PF, Chang PY and Kuo KN: A comparison of aspirin and clopidogrel with or without proton pump inhibitors for the secondary prevention of cardiovascular events in patients at high risk for gastrointestinal bleeding. Clin Ther 31: 2038-2047, 2009.

11. Liberati A, Altman DG, Tetzlaff J, Mulrow C, Gøtzsche PC, Ioannidis JP, Clarke M, Devereaux PJ, Kleijnen J and Moher D: The PRISMA statement for reporting systematic reviews and meta-analyses of studies that evaluate healthcare interventions: Explanation and elaboration. BMJ 339 (jul21 1): b2700, 2009.

12. Sterne JAC, Higgins JPT and Reeves BC: A Cochrane Risk of Bias Assessment Tool: for Non-Randomized Studies of Interventions (ACROBAT-NRSI). Version 1.0.0, September 24, 2014. Retrieved from http://www.riskofbias.info. Accessed: May 21, 2015.

13. Higgins JPT, Thompson SG, Deeks JJ and Altman DG: Measuring inconsistency in meta-analyses. BMJ 327: 557-560, 2003.

14. Ma L, Elliott SN, Cirino G, Buret A, Ignarro LJ and Wallace JL: Platelets modulate gastric ulcer healing: Role of endostatin and vascular endothelial growth factor release. Proc Natl Acad Sci USA 98: 6470-6475, 2001.

15. Ng FH, Wong BC, Wong SY, Chen WH and Chang CM: Clopidogrel plus omeprazole compared with aspirin plus omeprazole for aspirin-induced symptomatic peptic ulcers/ erosions with low to moderate bleeding/re-bleeding risk - a single-blind, randomized controlled study. Aliment Pharmacol Ther 19: 359-365, 2004.

16. Hass WK, Easton JD, Adams HP Jr, Pryse-Phillips W, Molony BA, Anderson S and Kamm B; Ticlopidine Aspirin Stroke Study Group: A randomized trial comparing ticlopidine hydrochloride with aspirin for the prevention of stroke in high-risk patients. N Engl J Med 321: 501-507, 1989.

17. Savi P, Bernat A, Dumas A, Aït-Chek L and Herbert JM: Effect of aspirin and clopidogrel on platelet-dependent tissue factor expression in endothelial cells. Thromb Res 73: 117-124, 1994.

18. Kim KA, Park PW, Hong SJ and Park JY: The effect of CYP2C19 polymorphism on the pharmacokinetics and pharmacodynamics of clopidogrel: A possible mechanism for clopidogrel resistance. Clin Pharmacol Ther 84: 236-242, 2008.

19. Sibbing D, Morath T, Stegherr J, Braun S, Vogt W, Hadamitzky M, Schömig A, Kastrati A and von Beckerath N: Impact of proton pump inhibitors on the antiplatelet effects of clopidogrel. Thromb Haemost 101: 714-719, 2009.

20. Gilard M, Arnaud B, Cornily JC, Le Gal G, Lacut K, Le Calvez G, Mansourati J, Mottier D, Abgrall JF and Boschat J: Influence of omeprazole on the antiplatelet action of clopidogrel associated with aspirin: The randomized, double-blind OCLA (Omeprazole CLopidogrel Aspirin) study. J Am Coll Cardiol 51: 256-260, 2008.

21. Juurlink DN, Gomes T, Ko DT, Szmitko PE, Austin PC, Tu JV, Henry DA, Kopp A and Mamdani MM: A population-based study of the drug interaction between proton pump inhibitors and clopidogrel. CMAJ 180: 713-718, 2009.

22. Ho PM, Maddox TM, Wang L, Fihn SD, Jesse RL, Peterson ED and Rumsfeld JS: Risk of adverse outcomes associated with concomitant use of clopidogrel and proton pump inhibitors following acute coronary syndrome. JAMA 301: 937-944, 2009.

23. Depta JP and Bhatt DL: Omeprazole and clopidogrel: Should clinicians be worried? Cleve Clin J Med 77: 113-116, 2010.

24. Leontiadis GI, Sharma VK and Howden CW: Systematic review and meta-analysis: Enhanced efficacy of proton-pump inhibitor therapy for peptic ulcer bleeding in Asia - a post hoc analysis from the Cochrane Collaboration. Aliment Pharmacol Ther 21: 1055-1061, 2005.

This work is licensed under a Creative Commons Attribution-NonCommercial-NoDerivatives 4.0 International (CC BY-NC-ND 4.0) License. 